TRANSACTIONS OF THE

AMERICAN MATHEMATICAL SOCIETY

Volume 284. Number 1, July 1984

\title{
MEAN VALUE PROPERTIES OF THE LAPLACIAN VIA SPECTRAL THEORY
}

\author{
BY
}

ROBERT S. STRICHARTZ ${ }^{1}$

\begin{abstract}
Let $\phi\left(z^{2}\right)$ be an even entire function of temperate exponential type, $L$ a selfadjoint realization of $-\Delta+c(x)$, where $\Delta$ is the Laplace-Beltrami operator on a Riemannian manifold, and $\phi(L)$ the operator given by spectral theory. A PaleyWiener theorem on the support of $\phi(L)$ is proved, and is used to show that $L u=\lambda u$ on a suitable domain implies $\phi(L) u=\phi(\lambda) u$, as well as a generalization of Asgeirsson's theorem. A concrete realization of the operators $\phi(L)$ is given in the case of a compact Lie group or a noncompact symmetric space with complex isometry group.
\end{abstract}

1. Introduction. The mean value of a harmonic function on a sphere is equal to the value of the function at the center. More generally, if $\Delta u=\lambda u$ on $\mathbf{R}^{n}$ then $M_{r} u=a(r, \lambda) u$, where $M_{r}$ denotes the mean value operator

$$
M_{r} u(x)=\int_{S^{n-1}} u(x+r y) d \mu(y)
$$

where $d \mu(y)$ is Lebesgue measure on the sphere $S^{n-1}$ normalized to have total measure one. Here

$$
a(r, \lambda)=2^{n / 2-1} \Gamma(n / 2) J_{n / 2-1}(r \sqrt{-\lambda}) /(r \sqrt{-\lambda})^{n / 2-1} .
$$

Àsgeirsson's theorem [1] asserts that if $u(x, y)$ is a $C^{2}$ solution of $\Delta_{x} u=\Delta_{y} u$ on $\mathbf{R}^{n} \times \mathbf{R}^{n}$ then $M_{r}(x) u=M_{r}(y) u$, where $M_{r}(x)$ denotes the operator $M_{r}$ in the $x$ variables alone, and similarly for $M_{r}(y)$. Since $M_{r}$ is a function of the Laplacian, in fact

$$
M_{r}=2^{n / 2-1} \Gamma(n / 2) J_{n / 2-1}(r \sqrt{-\Delta}) /(r \sqrt{-\Delta})^{n / 2-1}
$$

and the resemblance to (1.1) is not a coincidence, this suggests that the above results should be special cases of a general heuristic principle which we can state as follows: an equation involving the Laplacian implies an analogous equation involving functions of the Laplacian.

On a certain level this principle is a tautology. If $L$ is any selfadjoint operator on a dense domain in some Hilbert space $\mathscr{H}$, and if $u$ in $\mathscr{H}$ is an eigenvector, $L u=\lambda u$, then $\phi(L) u=\phi(\lambda) u$ by the definition of $\phi(L)$. To obtain a nontrivial result we

Received by the editors August 17, 1983.

1980 Mathematics Subject Classification. Primary 31C12, 35P99; Secondary 43A85.

Key' words and phrases. Laplace-Beltrami operator, spectral theory, mean value theorem, Asgeirsson's theorem, functions of the Laplacian, Paley-Wiener theorem, symmetric space, compact Lie group.

${ }^{1}$ Research supported in part by NSF Grant MCS-8002771. 
want to allow eigenvectors outside the Hilbert space, or in the case where $L$ is a differential operator on a manifold, we want to allow solutions of the eigenvalue equation on only part of the manifold. We accomplish this by assuming that $\phi\left(z^{2}\right)$ is an entire function of exponential type and proving in $\$ 2$ an abstract Paley-Wiener theorem that shows that $\phi(\Delta)$ is a compactly supported operator for a general Laplacian $\Delta$. This is applied to obtain an abstract mean value theorem in $\S 3$ and an abstract Àsgeirsson's theorem in \$4. Earlier.generalizations of Àsgeirsson's theorem due to Helgason may be found in [5, 6 and 8 ].

In order to give some concrete meaning to our results it is necessary to be able to recognize operators which are functions of the Laplacian. In Euclidean space it is easy to show that these are exactly the radial convolution operators, and an analogous statement is true on rank-one symmetric spaces (two-point homogeneous spaces). In $\$ 5$ we point out that it is possible to describe concretely the functions of the Laplacian on a compact Lie group or a noncompact symmetric space $G / K$, where $G$ is a complex Lie group. These results are not really new. For $G / K$ with $G$ complex a well-known formula of Harish-Chandra shows that any spherical transform can be computed by means of a Euclidean Fourier transform (this is used in Flensted-Jensen [3] in the proof of Theorem 3.1, for example). An analogous result on compact Lie groups was established in [10]. Also, a different approach to these results is implicitly contained in a recent paper of Helgason [8] - in fact, the present paper originated in an attempt to better understand some of the themes in Helgason's paper.

2. An abstract Paley-Wiener theorem. Let $M$ be a $C^{\infty}$ manifold without boundary with Riemannian metric, and denote by $d \mu$ the associated measure and $\Delta$ the Laplace-Beltrami operator. We consider any operator $L$ defined by

$$
L u=(-\Delta+c(x)) u
$$

on a dense domain $D(L)$ in $L^{2}(M, d \mu)$ containing $C_{\text {com }}^{\infty}(M)$, where $c(x)$ is a real-valued $C^{\infty}$ function on $M$ such that $L$ is selfadjoint on $D(L)$.

A function $\phi: \mathbf{C} \rightarrow \mathbf{C}$ is said to be an entire function of temperate exponential type $A$ if it is entire and satisfies an estimate

$$
|\phi(z)| \leqslant c(1+|z|)^{N} e^{A|\operatorname{Im} z|}
$$

for some constants $c$ and $N$ for all complex $z$. Now for a general measurable function $\phi$ defined on the spectrum of $L$, we can define $\phi(L)$ by the spectral theorem

$$
\phi(L)=\int \phi(t) d E_{t},
$$

where $E_{t}$ is the spectral projection associated with $L$, and the domain of $\phi(L)$ is suitably restricted. The abstract Paley-Wiener theorem asserts that if $\phi\left(z^{2}\right)$ is an even entire function of temperate exponential type $A$ then the kernel of $\phi(L)$ is supported in the $A$-neighborhood of the diagonal. To make this more precise we define the distance to the boundary of a point $x$ in $M$ to be the infimum of the lengths of all geodesic rays starting at $x$ and continued as far as possible. 
THEOREM 2.1. Let $L$ be as above and let $\phi\left(z^{2}\right)$ be an even entire function of temperate exponential type $A$. Let $x_{0}$ be any point in $M$ whose distance to the boundary exceeds $A$. Then there exists a distribution $T$ supported in the closed ball $B_{A}\left(x_{0}\right)$ of radius $A$ about $x_{0}$ such that $\phi(L) u\left(x_{0}\right)=\langle T, u\rangle$ for all $u \in C_{\mathrm{com}}^{\infty}(M)$.

Proof. The idea of the proof is to invoke the theory of hyperbolic partial differential equations to establish the conclusion for the special case $\phi(z)=\cos t \sqrt{z}$, $|t| \leqslant A$, and then use the Paley-Wiener theorem in $\mathbf{R}$ to pass to the general case.

Indeed, the assumptions on $L$ imply that the operator $\partial^{2} / \partial t^{2}+L$ is strictly hyperbolic, and the bicharacteristic flow associated with this operator coincides with the geodesic flow on the cotangent bundle of $M$. It follows from the theory of hyperbolic equations (see Chernoff [2]) that for each $t$ with $0 \leqslant t \leqslant A$ there exists a distribution $h_{t}$ supported in $B_{A}\left(x_{0}\right)$ such that $\cos t \sqrt{L} u\left(x_{0}\right)=\left\langle h_{t}, u\right\rangle$ for $u \in$ $C_{\text {com }}^{\infty}(M)$ and $\left\langle h_{t}, u\right\rangle$ depends smoothly on $t$. On the other hand, the Paley-Wiener theorem on $\mathbf{R}$ implies

$$
\phi\left(z^{2}\right)=\int_{0}^{A} \cos t z \psi(t) d t
$$

from some distribution $\psi$ supported in $[0, A]$. Thus we obtain

$$
\begin{aligned}
\phi(L) u\left(x_{0}\right) & =\int_{0}^{A} \cos t \sqrt{L} u\left(x_{0}\right) \psi(t) d t \\
& =\int_{0}^{A}\left\langle h_{t}, u\right\rangle \psi(t) d t=\langle T, u\rangle,
\end{aligned}
$$

where $T=\int_{0}^{A} h_{t} \psi(t) d t$ is supported in $B_{A}\left(x_{0}\right)$. Q.E.D.

REMARK. It would be interesting to know whether there is a converse to the theorem.

\section{An abstract mean value theorem.}

THEOREM 3.1. Let $L, \phi$ and $x_{0}$ be as in Theorem 2.1. Suppose $u$ satisfies the eigenvalue equation

$$
L u=\lambda u
$$

in a neighborhood of $B_{A}\left(x_{0}\right)$, for some complex $\lambda$. Then

$$
\phi(L) u\left(x_{0}\right)=\phi(\lambda) u\left(x_{0}\right) .
$$

In particular, if the manifold is complete and $u$ satisfies (3.1) on the whole manifold, then it satisfies (3.2) on the whole manifold.

Proof. Since $L$ is elliptic $u$ must be $C^{\infty}$ in a neighborhood of $B_{A}\left(x_{0}\right)$, so by multiplying by an appropriate cut-off function we may obtain a function $v$ in $C_{\mathrm{com}}^{\infty}(M)$ such that $v=u$ on $B_{A+\varepsilon}\left(x_{0}\right)$ for sufficiently small $\varepsilon$, and then

$$
(L-\lambda) v=g \text { where } g=0 \text { on } B_{A+\varepsilon}\left(x_{0}\right) .
$$

The idea of the proof is to apply the operator

$$
\phi_{1}(L)=\frac{\phi(L)-\phi(\lambda)}{L-\lambda}
$$


to both sides of (3.3). It is easy to see that

$$
\phi_{1}\left(z^{2}\right)=\frac{\phi\left(z^{2}\right)-\phi(\lambda)}{z^{2}-\lambda}
$$

is also an even entire function of temperate exponential type $A$, so Theorem 2.1 applies to $\phi_{1}(L)$ and we conclude

$$
(\phi(L)-\phi(\lambda)) u\left(x_{0}\right)=\phi_{1}(L) g(x)=\langle T, g\rangle=0
$$

since $T$ is supported in $B_{A}\left(x_{0}\right)$ and $g$ vanishes on $B_{A+\varepsilon}\left(x_{0}\right)$. Q.E.D.

In many cases it suffices to assume that $L u=\lambda u$ on the interior of $B_{A}\left(x_{0}\right)$, and that $u$ is sufficiently regular on $B_{A}\left(x_{0}\right)$ so that $\phi(L) u\left(x_{0}\right)$ is well defined. Such an improvement may be obtained by approximating $\phi$ by entire functions of temperate exponential type $A-\varepsilon$. We will not attempt to formulate a general result, but we mention one example: if $u$ is continuous on $B_{A}\left(x_{0}\right)$ and harmonic in the interior on $\mathbf{R}^{n}$, then the theorem implies $M_{r} u\left(x_{0}\right)=u\left(x_{0}\right)$ for all $r<A$. But then we may take the limit as $r \rightarrow A$ to obtain $M_{A} u\left(x_{0}\right)=u\left(x_{0}\right)$, the usual mean value theorem. The same result holds on any rank-one symmetric space since we know that $M_{r}=\phi(-\Delta)$, and $\phi\left(z^{2}\right)$ is an even entire function of temperate exponential type $r$ because of the Paley-Wiener theorem for symmetric spaces [7].

4. An abstract Àsgeirsson's theorem. Let $M_{1}$ and $M_{2}$ be two Riemannian manifolds with distances $d_{1}$ and $d_{2}$ and operators $L_{1}$ and $L_{2}$ as before. For a point $\left(x_{0}, y_{0}\right) \in$ $M_{1} \times M_{2}$ we let

$$
D_{A}\left(x_{0}, y_{0}\right)=\left\{(x, y) \in M_{1} \times M_{2}: d_{1}\left(x, x_{0}\right)+d_{2}\left(y, y_{0}\right) \leqslant A\right\}
$$

be the diamond-shaped domain centered at $\left(x_{0}, y_{0}\right)$.

THEOREM 4.1. Let $\left(x_{0}, y_{0}\right)$ be a point in $M_{1} \times M_{2}$ such that the sum of the distances to the boundary for $x_{0}$ in $M_{1}$ and $y_{0}$ in $M_{2}$ exceeds $A$, and let $\phi\left(z^{2}\right)$ be an even entire function of temperate exponential type $A$. If $u$ is a $C^{\infty}$ solution of

$$
L_{1} u=L_{2} u
$$

in a neighborhood of $D_{A}\left(x_{0}, y_{0}\right)$ in $M_{1} \times M_{2}$, then

$$
\phi\left(L_{1}\right) u\left(x_{0}, y_{0}\right)=\phi\left(L_{2}\right) u\left(x_{0}, y_{0}\right) .
$$

Proof. Let $v$ be in $C_{\text {com }}^{\infty}\left(M_{1} \times M_{2}\right)$ with $v=u$ on $D_{A+\varepsilon}\left(x_{0}, y_{0}\right)$, so

$$
\left(L_{1}-L_{2}\right) v=g \text { with } g=0 \text { on } D_{A+\varepsilon}\left(x_{0}, y_{0}\right) \text {. }
$$

To complete the proof as in Theorem 3.1 we need to show that the operator

$$
\frac{\phi\left(L_{1}\right)-\phi\left(L_{2}\right)}{L_{1}-L_{2}}
$$

at $\left(x_{0}, y_{0}\right)$ is given by a distribution with support in $D_{A}\left(x_{0}, y_{0}\right)$. We will do this by proving

$$
\frac{\phi\left(L_{1}\right)-\phi\left(L_{2}\right)}{L_{1}-L_{2}}=\int_{\left|r_{1}\right|+\left|t_{2}\right| \leqslant A} \cos t_{1} \sqrt{L_{1}} \cos t_{2} \sqrt{L_{2}} \psi(t) d t
$$


where $\psi$ is a distribution in $\mathbf{R}^{2}$ supported in $\left|t_{1}\right|+\left|t_{2}\right| \leqslant A$. As in the proof of Theorem 2.1, the operator on the right side of (4.4) at the point $\left(x_{0}, y_{0}\right)$ is given by a distribution with support in $D_{A}\left(x_{0}, y_{0}\right)$, and the existence of $\psi$ in (4.4) is a consequence of a variant of the Paley-Wiener theorem in $\mathbf{R}^{2}$ if we can show that

$$
\phi_{1}\left(z_{1}^{2}, z_{2}^{2}\right)=\frac{\phi\left(z_{1}^{2}\right)-\phi\left(z_{2}^{2}\right)}{z_{1}^{2}-z_{2}^{2}}
$$

is an entire function, even in both variables separately, which satisfies a growth condition

$$
\left|\phi_{1}\left(z_{1}^{2}, z_{2}^{2}\right)\right| \leqslant c\left(1+\left|z_{1}\right|^{2}+\left|z_{2}\right|^{2}\right)^{N} e^{A \max \left(\left|\operatorname{Im} z_{1}\right|,\left|\operatorname{Im} z_{2}\right|\right)}
$$

for some $c$ and $N$. These conditions are all obvious except for the estimate (4.5) in the region $\left|z_{1}^{2}-z_{2}^{2}\right| \leqslant 1$. Now we may write

$$
\phi_{1}\left(z_{1}^{2}, z_{2}^{2}\right)=\frac{1}{z_{1}^{2}-z_{2}^{2}} \int_{z_{1}^{2}}^{z_{2}^{2}} \phi^{\prime}(\zeta) d \zeta,
$$

and we use the fact that the derivative of $\phi\left(z^{2}\right)$ is also an entire function of temperate exponential type $A$ to estimate

$$
\left|\phi^{\prime}\left(z^{2}\right)\right| \leqslant c(1+|z|)^{N} e^{A|\operatorname{Im} z|} \text {. }
$$

We choose the curve joining $z_{1}^{2}$ and $z_{2}^{2}$ in (4.6) by squaring the straight-line segment joining $z_{1}$ and $\pm z_{2}$, choosing the \pm so that $\operatorname{Im} z_{1}$ and $\pm \operatorname{Im} z_{2}$ have the same sign. In this way $\zeta=z^{2}$ with $|\operatorname{Im} z| \leqslant \max \left(\left|\operatorname{Im} z_{1}\right|,\left|\operatorname{Im} z_{2}\right|\right)$ along the curve and the length of the curve is comparable to $z_{1}^{2}-z_{2}^{2}$, so the estimate (4.7) plugged into (4.6) will imply (4.5). Q.E.D.

Because the differential equation (4.1) is not hypoelliptic, the hypothesis that $u$ be $C^{\infty}$ is unnatural, and we would like to relax it. This is possible if we make further assumptions on $\phi\left(L_{1}\right)$ and $\phi\left(L_{2}\right)$ so that (4.2) makes sense. For example, if the distributions giving $\phi\left(L_{1}\right)$ and $\phi\left(L_{2}\right)$ are measures, then the result remains true if $u$ is merely continuous. Notice that we can guarantee $\phi(L) u\left(x_{0}\right)=\int_{M} u(x) d \mu(x)$, where $\mu$ is a finite measure depending continuously on $x_{0}$ if we assume

$$
|\phi(x)| \leqslant c(1+|x|)^{-r}
$$

for some $r>n / 2$ and $x$ in the spectrum of $L$, where $n$ is the dimension of the manifold, in addition to the hypothesis that $\phi\left(z^{2}\right)$ is an even entire function of temperate exponential type. To see this we observe that (4.8) implies $\phi(L)$ is a bounded operator from $L^{2}$ to the Sobolev space $H_{\mathrm{loc}}^{r}$, and by the Sobolev embedding theorem $H_{\mathrm{loc}}^{r}$ is contained in the continuous functions. Since we already know that $\phi(L)$ is represented by a distribution of compact support, the distribution must be a finite measure by the Riesz representation theorem.

COROLlARY 4.2. If in addition to the hypotheses of the theorem we assume $\phi\left(L_{1}\right)$ and $\phi\left(L_{2}\right)$ are represented by finite measures depending continuously on the point, then the conclusion remains true if we assume merely that $u$ is continuous and satisfies (4.1) in the distribution sense. 
Proof. The idea is to regularize $u$ by operators which commute with $L_{1}$ and $L_{2}$. We choose an "approximate identity" $\psi_{\varepsilon}(t)$ which is rapidly decreasing for real $t$, such that $\psi_{\varepsilon}\left(z^{2}\right)$ is an even entire function of temperate exponential type $\varepsilon$, and such that $\psi_{\varepsilon}(t) \rightarrow 1$ as $\varepsilon \rightarrow 0$ uniformly on compact sets. Then $\psi_{\varepsilon}\left(L_{1}\right) \psi_{\varepsilon}\left(L_{2}\right) u$ will be a $C^{\infty}$ function satisfying (4.1) on a neighborhood of $D_{A}\left(x_{0}, y_{0}\right)$ if $\varepsilon$ is small enough. By the theorem

$$
\phi\left(L_{1}\right) \psi_{\varepsilon}\left(L_{1}\right) \psi_{\varepsilon}\left(L_{2}\right) u=\phi\left(L_{2}\right) \psi_{\varepsilon}\left(L_{1}\right) \psi_{\varepsilon}\left(L_{2}\right) u\left(x_{0}, y_{0}\right)
$$

hence

$$
\psi_{\varepsilon}\left(L_{1}\right) \psi_{\varepsilon}\left(L_{2}\right) \phi\left(L_{1}\right) u\left(x_{0}, y_{0}\right)=\psi_{\varepsilon}\left(L_{1}\right) \psi_{\varepsilon}\left(L_{2}\right) \phi\left(L_{2}\right) u\left(x_{0}, y_{0}\right)
$$

since all the operators commute, and the same will be true on a sufficiently small neighborhood of $\left(x_{0}, y_{0}\right)$. Now $\psi_{\varepsilon}\left(L_{1}\right) \psi_{\varepsilon}\left(L_{2}\right)$ is an $L^{2}$ approximate identity, so we may take the limit as $\varepsilon \rightarrow 0$ and obtain $\phi\left(L_{1}\right) u(x, y)=\phi\left(L_{2}\right) u(x, y)$ in the $L^{2}$ sense in a neighborhood of $\left(x_{0}, y_{0}\right)$. But since these functions are continuous by hypothesis, the identity must hold pointwise as well. Q.E.D.

5. Functions of the Laplacian on certain symmetric spaces. In $\mathbf{R}^{n}$ we know that functions of the Laplacian are convolution operators with radial kernels. This can be stated quantitatively as $\phi(-\Delta) u=H_{\phi} * u$, where

$$
H_{\phi}(x)=(2 \pi)^{n / 2} \int_{0}^{\infty} \phi\left(s^{2}\right) \frac{J_{n / 2-1}(s|x|)}{(s|x|)^{n / 2-1}} s^{n-1} d s
$$

(see [9]) or qualitatively as follows: the class of operators $\phi(-\Delta)$ with $\phi\left(z^{2}\right)$ an even entire function of temperate exponential type $A$ is equal to the class of operators $u \rightarrow H * u$, where $H$ is radial distribution supported in $|x| \leqslant A$. As we mentioned before, the qualitative result remains valid in a rank-one symmetric space. Here we wish to point out that analogous results, both quantitative and qualitative, hold for compact Lie groups and for symmetric spaces of the noncompact type $G / K$ with $G$ complex.

Let $G$ be a complex connected semisimple Lie group with finite center, $K$ a maximal compact subgroup and $X=G / K$ the symmetric space with Riemannian metric induced by the Killing form $B$ on the Lie algebra $g$ of $G$. Let $g=\mathfrak{f}+\mathfrak{p}$ be the corresponding Cartan decomposition, and Exp: $\mathfrak{p} \rightarrow X$ the exponential map. Let Log: $X \rightarrow \mathfrak{p}$ denote the inverse and $J$ the Jacobian of Exp, so we have

$$
\int_{G / K} f(x) d x=\int_{\mathfrak{p}} f(\operatorname{Exp} Y) J(Y) d Y .
$$

Because $G$ is complex, $J(Y)$ has a nonvanishing $C^{\infty}$ square-root, in fact

$$
J(Y)^{1 / 2}=\prod_{\alpha \in \Sigma^{+}} \frac{\sinh \alpha(H)}{\alpha(H)},
$$

where $H$ is conjugate to $Y$ and lies in a maximal abelian subspace $\mathfrak{A} \subseteq \mathfrak{p}$, and $\Sigma^{+}$ denotes the set of positive restricted roots not counting multiplicity (each has multiplicity two). 
Let $c_{0}$ denote the constant $\frac{1}{48} \operatorname{dim} X$ (this is also equal to $B(\rho, \rho)$, where $\rho=$ $\left.\Sigma_{\alpha \in \Sigma^{+}} \alpha\right)$ and let $L=-\Delta-c_{0}$. With this choice of $c_{0}$ the spectrum of $L$ is exactly the positive real axis.

THEOREM 5.1. Let $\phi\left(z^{2}\right)$ be an even entire function of temperate exponential type A. Then $\phi(L)$ is the operator of convolution on $X$ with the $K$-invariant kernel $J^{-1 / 2}(\log x) H_{\phi}(\log x)$, where $H_{\phi}$ is given by (5.1) with $n=\operatorname{dim} X$.

PROOF. This is almost an immediate consequence of Harish-Chandra's formula

$$
\Phi_{\lambda}(\operatorname{Exp} X)=J^{-1 / 2}(X) \int_{K} e^{i \lambda(\operatorname{ad} k X)} d k
$$

for the spherical function, where $\lambda$ is the parameter in $\mathfrak{A}^{*}$ (or even $\mathfrak{p}^{*}$ ) and the Haar measure on $K$ is normalized to have total mass one [4, §14]. The choice of $L$ is such that $L \Phi_{\lambda}=|\lambda|^{2} \Phi_{\lambda}$. Now we know that $\phi(L)$ is a convolution operator with $K$-invariant kernel, so it suffices to verify that

$$
\left\langle J^{-1 / 2}(\log x) H_{\phi}(\log x), \Phi_{\lambda}(x)\right\rangle=\phi\left(|\lambda|^{2}\right) .
$$

But by (5.2) and (5.3)

$$
\begin{aligned}
\left\langle J^{-1 / 2}(\log x) H_{\phi}(\log x)\right. & \left., \Phi_{\lambda}(x)\right\rangle=\left\langle H_{\phi}(X), \int_{K} e^{i \lambda(\operatorname{ad} k X)} d k\right\rangle \\
= & \int_{K}\left\langle H_{\phi}(X), e^{i \lambda(\operatorname{ad} k X)}\right\rangle d k=\int_{K} \phi\left(|\operatorname{ad} k \lambda|^{2}\right) d k=\phi\left(|\lambda|^{2}\right)
\end{aligned}
$$

since $H_{\phi}$ is the radial Euclidean Fourier transform of $\phi$.

We can also give a different proof based on a recent result of Helgason [8].

Because $L$ is $G$-invariant we know that $\phi(L)$ must be convolution with the $K$-invariant kernel $\phi(L) \delta$, where $\delta$ denotes the $\delta$-distribution at the origin. Now Helgason [8, Theorems 4.3 and 4.4] shows that

$$
\left\langle\frac{\sinh t \sqrt{L}}{\sqrt{L}} \delta, f\right\rangle=\left\langle\frac{\sinh t \sqrt{-\Delta}}{\sqrt{-\Delta}} \delta, J^{1 / 2} \cdot f \circ \operatorname{Exp}\right\rangle
$$

for all $t$ and $f \in C_{\mathrm{com}}^{\infty}(X)$, where on the right side of (5.4) we have the distribution $\sin t \sqrt{-\Delta} \delta / \sqrt{-\Delta}$ on $\mathfrak{p}$ with $\Delta$ the Laplacian on $\mathfrak{p}$ and $\delta$ the $\delta$-distribution at the origin of $\mathfrak{p}$. Now if we differentiate this identity with respect to $t$, multiply by the Fourier transform of $\phi\left(t^{2}\right)$ and integrate, we obtain

$$
\langle\phi(L) \delta, f\rangle=\left\langle\phi(-\Delta) \delta, J^{1 / 2} \cdot f \circ \operatorname{Exp}\right\rangle .
$$

From the Euclidean theory of $\mathfrak{p}$ we know $\phi(-\Delta) \delta=H_{\phi}$, and so

$$
\langle\phi(L) \delta, f\rangle=\left\langle H_{\phi}, J^{1 / 2} \cdot f \circ \operatorname{Exp}\right\rangle,
$$

and finally using (5.2) to transfer the pairing from $\mathfrak{p}$ to $X$ we obtain

$$
\langle\phi(L) \delta, f\rangle=\left\langle\left(J^{-1 / 2} \circ \log \right)\left(H_{\phi} \circ \log \right), f\right\rangle \text {. Q.E.D. }
$$

REMARK. It would be interesting to understand the relationship between $\phi(L)$ and $\phi(-\Delta)$ for the Laplacian on $p$ in the case of a general noncompact symmetric space. The example of hyperbolic space of odd dimension, $X=S O(n, 1) / S O(n)$ with $n$ 
odd, gives an inkling of what might be expected. In this case the Euclidean formula (5.1) can be written (using recursion relations for the Bessel functions) $\phi(-\Delta) u=$ $H_{\phi} * u$ with

$$
H_{\phi}(x)=2\left(\frac{-2 \pi}{t} \frac{\partial}{\partial t}\right)^{n / 2-1} \int_{0}^{\infty} \phi\left(s^{2}\right) \cos s t d s,
$$

where $t=|x|$ is the Euclidean distance to the origin, while the non-Euclidean formula can be written $\phi(L)=\tilde{H}_{\phi} * u$ with

$$
\tilde{H}_{\phi}(x)=2\left(\frac{-2 \pi}{\sin h t} \frac{\partial}{\partial t}\right)^{(n-1) / 2} \int_{0}^{\infty} \phi\left(s^{2}\right) \cos s t d s,
$$

where $t$ is the hyperbolic distance to the origin. This is easily derived from the explicit expression for the spherical functions in Takahashi [11].

Corollary 5.2. A K-invariant distribution $T$ on $X$ of compact support defines by convolution an operator of the form $\phi(L)$ for some even entire function $\phi\left(z^{2}\right)$ of temperate exponential type if and only if $T=\left(J^{-1 / 2} \circ \log \right) H d x$, where $H$ is a radial distribution (depending only on the distance to the origin) of compact support.

Proof. By the Euclidean Paley-Wiener theorem there is a one-to-one correspondence between distributions $H_{\phi}$ and radial distributions of compact support on $\mathfrak{p}$. If $H_{\phi}$ happens to be a locally integrable function then the form of $T=\phi(L) \delta$ in the theorem is

$$
\langle T, f\rangle=\int_{X} J^{-1 / 2}(\log x) H_{\phi}(\log x) f(x) d x
$$

and $H_{\phi}(\log x)=H(x)$ depends only on the distance to the origin. In general we can write formally $T=\left(J^{-1 / 2} \circ \log \right) H d x$, where $H(x)=H_{\phi}(\log x)$ is a radial distribution. Q.E.D.

There are analogous results for compact semisimple Lie groups $K$, which are the dual symmetric spaces to $G / K$ for $G$ complex. We consider on $K$ the Riemannian metric induced by the Killing form $B$ on the Lie algebra $f$ and the associated Laplace-Beltrami operator $\Delta$ (note that this is a different normalization from the one for $G / K$ since the full isometry group of $K$ as a symmetric space is $K \times K$, and so some of the formulas in the compact case will differ from those in the noncompact case by a factor of 2). Let Exp: $\mathfrak{f} \rightarrow K$ be the exponential mapping and $R$ the injectivity radius, the largest number such that Exp is a diffeomorphism of the open ball of radius $R$ about the origin to its image. We denote by Log the inverse of Exp restricted to this ball, and $J$ the Jacobian of Exp. Again we have the integral formula

$$
\int_{K} f(x) d x=\int_{B_{R}(0)} f(\operatorname{Exp} Y) J(Y) d Y
$$


if $f$ is supported in $B_{R}(0)$, and $J(Y)$ has a nonvanishing $C^{\infty}$ square root in $B_{R}(0)$,

$$
J(Y)^{1 / 2}=\prod_{\alpha \in \Sigma^{+}} \frac{\sin \alpha(H)}{\alpha(H)},
$$

where $H$ is conjugate to $Y$ in $\mathfrak{A}$ and $\Sigma^{+}$denotes the set of positive roots. In this case the constant $c_{0}=\frac{1}{24} \operatorname{dim} X$ and we set $L=-\Delta+c_{0}$. The analogues of the previous theorem and its corollary are as follows.

THEOREM 5.3. Let $\phi\left(z^{2}\right)$ be an even entire function of temperate exponential type A, with $A<R$. Then $\phi(L)$ is the operator of convolution on $K$ with the kernel $J^{-1 / 2}(\log x) H_{\phi}(\log x)$, where $H_{\phi}$ is a distribution supported in $B_{A}(e)$ given by (5.1) with $n=\operatorname{dim} K$.

Corollary 5.4. $A$ distribution $T$ on $K$ with support in $B_{A}(e)$ for $A<R$ defines by convolution an operator of the form $\phi(L)$ for some even entire function $\phi\left(z^{2}\right)$ of temperate exponential type $A$ if and only if $T=\left(J^{-1 / 2} \circ \log \right) H d x$ for $H$ a radial distribution supported in $B_{A}(e)$.

Theorem 5.3 is essentially proved in Lemma 1 of [10]. It can also be derived from Theorems 4.5 and 4.6 of Helgason [8]. It is possible to formulate the result in such a way as to eliminate the condition $A<R$ (see [10]). The corollary follows as before. Finally, combining the results of this section with Theorem 3.1 and Corollary 4.2 , we obtain

THEOREM 5.5. Let $u$ be $a$ solution of $\Delta u=\lambda u$ in the interior of $B_{r}\left(x_{0}\right)$ and continuous in $B_{r}\left(x_{0}\right)$ for some point $x_{0}$ in $X$ or $K$. Then $M_{r} u\left(x_{0}\right)=a\left(r, \lambda \pm c_{0}\right) u\left(x_{0}\right)$, where $a$ is given by (1.1) and

$$
M_{r} u\left(x_{0}\right)=\int_{S^{n-1}} u\left((\operatorname{Exp} r y) x_{0}\right) J^{1 / 2}(r y) d \mu(y),
$$

where $d \mu$ is the normalized Lebesgue measure on the unit sphere in $\mathfrak{p}$, respectively $\mathfrak{f}$, and the plus sign is taken for $X$ and the minus sign for $K$.

THEOREM 5.6. Let $u$ be a continuous function in a neighborhood of a diamond-shaped domain $D_{r}\left(x_{0}, y_{0}\right)$ in $X \times X$ or $K \times K$, which satisfies $\Delta_{x} u=\Delta_{y} u$ in the distribution sense. Then

$$
M_{r}(x) u\left(x_{0}, y_{0}\right)=M_{r}(y) u\left(x_{0}, y_{0}\right)
$$

\section{REFERENCES}

1. L. Àsgeirsson, Über eine Mitterwertseigenshaft von Lösungen homogener linearer partieller Differentialgleichung 2. Ordnung mit Konstanten Koefficienten, Math. Ann. 113 (1936), 321-346.

2. P. R. Chernoff, Essential self-adjointness of powers of generators of hyperbolic equations, J. Functional Anal. 12 (1973), 401-414.

3. M. Flensted-Jensen, Spherical functions on a real semisimple Lie group. A method of reduction to the complex case, J. Functional Anal. 30 (1978), 106-146.

4. Harish-Chandra, Spherical functions on a semisimple Lie group. I, Amer. J. Math. 80 (1958), $241-310$. 
5. S. Helgason, Partial differential equations on Lie groups, Scand. Math. Congress XIII, Helsinki, 1957, pp. 110-115.

6. , Differential operators on homogeneous spaces, Acta Math. 102 (1959), 239-299.

7. __ Analysis on Lie groups and homogeneous spaces, C.B.M.S. Regional Conf. Ser. in Math., vol. 14, Amer. Math. Soc., Providence, R. I., 1972.

8. Wave equations on homogeneous spaces, preprint.

9. E. M. Stein and G. Weiss, Introduction to Fourier analysis on Euclidean spaces, Princeton Univ. Press, Princeton, N. J., 1971.

10. R. S. Strichartz, Multiplier transformations on compact Lie groups and algebras, Trans. Amer. Math. Soc. 193 (1974), 99-110.

11. R. Takahashi, Sur les réprésentations unitaire des groups de Lorentz généralizés, Buil. Soc. Math. France 91 (1963), 289-433.

Department of Mathematics, Cornell University, Ithaca, New York 14853 